\title{
Assessing psychache as a suicide risk variable: Data with the Portuguese version of the psychache scale
}

\author{
Rui C. Campos ${ }^{\mathrm{a}}$, Ronald R. Holden ${ }^{\mathrm{b}}$, and Margarida Gomes ${ }^{\mathrm{a}}$ \\ ${ }^{a}$ Department of Psychology, School of Social Sciences, University of Évora, Évora, Portugal; 'Department of Psychology, Queen's \\ University, Kingston, Ontario, Canada
}

\begin{abstract}
Several studies have consistently related psychological pain to suicide risk. Psychache, according to Shneidman's perspective and measured by the Psychache Scale has been confirmed as an important variable in risk prediction. In the present study, we evaluated psychache as a construct related to suicide risk using data obtained with the Portuguese version of the Psychache Scale translated from the original English version. A community sample of 628 individuals responded to the Portuguese version of the Psychache Scale, the Suicidal Behavior Questionnaire-Revised, the CES-D Scale, the Beck Hopelessness Scale, and the Suicide Ideation Questionnaire. Results supported the unidimensional scoring of the Psychache scale, its ability to differentiate between individuals at-risk for suicide from individuals not at-risk, its relationship with different, but related, constructs and its ability to predict suicide ideation.
\end{abstract}

\section{KEYWORDS}

Psychache; suicide risk

variable; Psychache Scale

\section{Introduction}

Despite suicide ideation being systematically linked to suicide attempts (Brezo, Paris, Tremblay, Vitaro, Hébert, \& Turecki, 2007), individuals who have never attempted suicide and who are not current suicide ideators may still be at risk in that a significant percentage of individuals do not report suicide ideation and/or suicide intention before a first attempt (Flett, Hewitt, \& Heisel, 2014). Thus, assessing other risk factors may be an important procedure for clinicians even when patients do not report significant suicidality.

Several types of variables have been linked to suicide risk, in particular psychological variables. One of the most compelling psychological perspectives of suicide is that of Shneidman (1993). The focal construct of his model is psychological pain. Shneidman (1993, p. 145) asserted that "Suicide is caused by psychache". Shneidman (1993) defined psychache as an acute state of intolerable psychological pain derived from an introspective experience linked to negative emotions. Empirical evidence supports Shneidman's model and includes research on suicidal manifestations as a function of internal perturbation for several types of samples, including suicide attempters (Holden \& DeLisle, 2006) and university students (Troister, D'Agata, \& Holden, 2015). Consistent with the more popular Interpersonal Psychological Theory of suicide, Shneidman (1993) argued that the cause of this pain is frustrated basic psychological needs and considered that psychological pain is triggered by negative life events and is both the cause and the necessary condition for suicide to occur. All other affective states (e.g., depression, hopelessness), although related to suicide, are relevant only if this intense psychological pain is present.

Several studies, framed in a psychological understanding of suicide (e.g. Interpersonal Theory of Suicide, Beck's hopelessness perspective) have provided a contribution to the understanding of suicide risk (e.g., Joiner, 2005). Within this psychological approach, Flamenbaum and Holden (2007) have consistently related psychological pain to suicide risk confirming that psychache is an important variable in risk prediction. Psychache has differentiated between those persons reporting suicide ideas and those individuals who had formulated a suicide plan, with the first group presenting lower overall scores on the Psychache Scale (Johns \& Holden 1997). Research has

CONTACT: Rui C. Campos rcampos@uevora.pt Departamento de Psicologia, Escola de Ciências Sociais e Centro de Investigação em Educação e Psicologia (CIEP-UE), Universidade de Évora, Universidade de Évora, Apartado 94, 7002-554 Évora, Portugal.

(C) 2018 Taylor \& Francis Group, LLC 